\title{
Quantum coherence spectroscopy to measure dietary fat retention in the liver
}

\author{
Lucas Lindeboom, ${ }^{1,2,3}$ Robin A. de Graaf, ${ }^{4}$ Christine I. Nabuurs, ${ }^{1,2,3}$ Petronella A. van Ewijk, ${ }^{1}$ \\ Matthijs K.C. Hesselink, ${ }^{2}$ Joachim E. Wildberger, ${ }^{1}$ Patrick Schrauwen, ${ }^{1,2,3}$ \\ and Vera B. Schrauwen-Hinderling ${ }^{1,2,3}$ \\ Departments of Radiology, ${ }^{2}$ Human Biology and Human Movement Sciences, NUTRIM school for Nutrition and \\ Translational Research in Metabolism, Maastricht University Medical Center, Maastricht, Netherlands. ${ }^{3}$ Top Institute \\ Food and Nutrition, Wageningen, Netherlands. ${ }^{4}$ Department of Radiology and Biomedical Imaging, Magnetic Resonance \\ Research Center, Yale University School of Medicine, New Haven, Connecticut, USA.
}

The prevalence of fatty liver reaches alarming proportions. Fatty liver increases the risk for insulin resistance, cardiovascular disease, and nonalcoholic steatohepatitis (NASH). Although extensively studied in a preclinical setting, the lack of noninvasive methodologies hampers our understanding of which pathways promote hepatic fat accumulation in humans. Dietary fat retention is one of the pathways that may lead to fatty liver. The low (1.1\%) natural abundance (NA) of carbon-13 $\left({ }^{13} \mathrm{C}\right)$ allows use of ${ }^{13} \mathrm{C}$-enriched lipids for in vivo MR studies. Successful implementation of such methodology, however, is challenging due to low sensitivity of ${ }^{13} \mathrm{C}$-magnetic resonance spectroscopy $\left({ }^{13}\right.$ C-MRS). Here, we investigated the use of 1-dimensional gradient enhanced heteronuclear single quantum coherence (ge-HSQC) spectroscopy for the in vivo detection of hepatic ${ }^{1} \mathrm{H}-\left[{ }^{13} \mathrm{C}\right]$-lipid signals after a single high-fat meal with ${ }^{13} \mathrm{C}$-labeled fatty acids in 5 lean and 6 obese subjects. Postprandial retention of orally administered ${ }^{13} \mathrm{C}$-labeled fatty acids was significant $(P<0.01)$. Approximately $1.5 \%$ of the tracer was retained in the liver after 6 hours, and retention was similar in both groups $(P=0.92)$. Thus, a substantial part of the liver fat can originate directly from storage of mealderived fat. The ge-HSQC can be used to noninvasively reveal the contribution of dietary fat to the development of hepatic steatosis over time.

Conflict of interest: The authors declare that no conflict of interest exists.

Submitted: September 15, 2015 Accepted: July 19, 2016 Published: August 18, 2016

Reference information: JCl Insight. 2016;1(13):e84671. doi:10.1172/jici.nsight.84671.

\section{Introduction}

Proton magnetic resonance spectroscopy $\left({ }^{1} \mathrm{H}-\mathrm{MRS}\right)$ has offered the opportunity to noninvasively detect intraorgan lipids in nonadipose tissue, such as liver and muscle, so-called ectopic fat $(1,2)$. In fact, after the introduction and the widespread use of this technique, evidence has accumulated that, in humans, increased intrahepatic lipid content (IHL content) is a very common condition, which strongly increases the risk to develop type 2 diabetes mellitus (T2DM) $(3,4)$. Furthermore, a fatty liver also increases the risk for hypertriglyceridemia, cardiovascular disease, and nonalcoholic steatohepatitis (NASH) (5-7). Fatty liver disease is increasingly considered a major threat to metabolic health in overweight populations.

Despite the general appreciation of the unhealthy consequences of a fatty liver, the factors that contribute to hepatic fat accumulation in humans are still far from understood and mainly rely on studies performed in animal models. De novo lipogenesis, excessive (adipose tissue) lipolysis, and dietary fat retention are the pathways that contribute to liver fat, but their relative contribution in humans is still unknown. In fact, due to the lack of noninvasive methodologies, these pathways could so far only be estimated in patients scheduled for liver biopsies (8) and may therefore not necessarily reflect normal physiology. Clearly, tools are needed to investigate de novo lipogenesis, adipose tissue lipolysis, and dietary fat retention in human volunteers, including examining the effect of intervention studies.

Although ${ }^{1} \mathrm{H}-\mathrm{MRS}$ can be used to measure total IHL content in vivo, this method does not yield information on the relative contribution of dietary fat retention to liver fat accumulation. As an alternative to magnetic resonance (MR), a fluorione-18 $\left({ }^{18} \mathrm{~F}\right)$ PET-tracer, $14-\left[{ }^{18} \mathrm{~F}\right]$ fluoro-6-thia-heptadecanoatecan $\left({ }^{18} \mathrm{FTHA}\right)$, can be used in humans to noninvasively determine organ-specific dietary fatty acid uptake after a meal (9). However, next to the disadvantage of the exposure to radioactivity, the ${ }^{18}$ FTHA tracer is trapped inside the hepatocyte upon entrance. Hence, this tracer only provides information on uptake. Here, we investigated 
A Conventional ge-HMQC

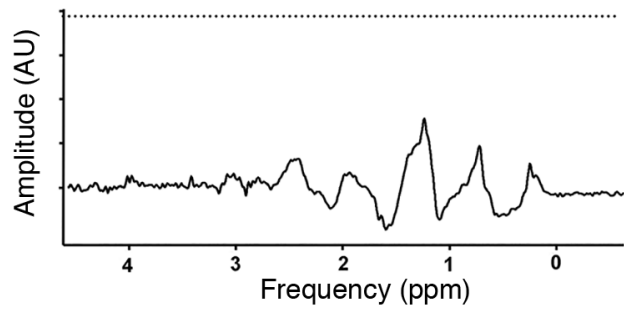

C 1D ge-HMQC

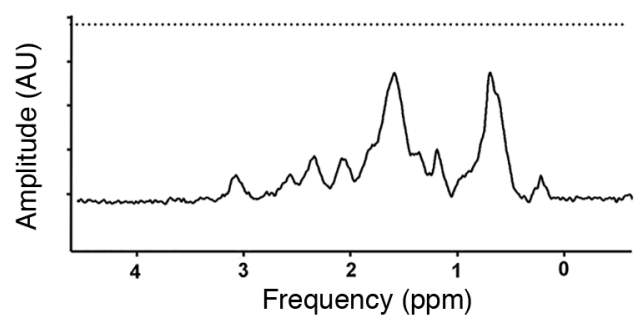

B Conventional ge-HSQC

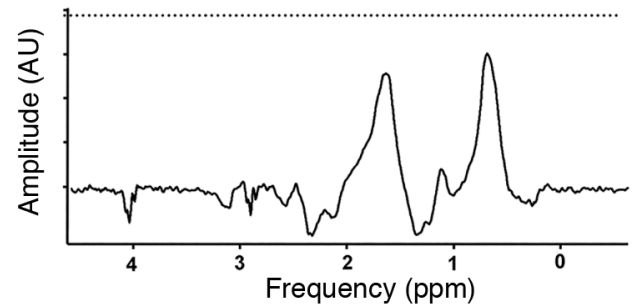

D $1 \mathrm{D} \mathrm{ge}-\mathrm{HSQC}$

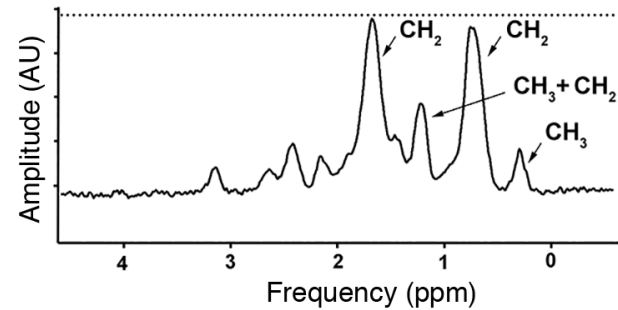

Figure 1. Spectra acquired from the phantom containing naturally abundant ${ }^{13} \mathbf{C}$ Intralipid. (A and B) The result from the conventional quantum coherence sequences. In both cases, the ${ }^{13} \mathrm{C}$ chemical shift evolution during the $\mathrm{t}_{1}$ period resulted in phase distortions in the spectrum. Addition of the ${ }^{13} \mathrm{C}$ inversion pulse led to nonphase distorted lipid spectra, in which both $\mathrm{CH}_{2}$ and $\mathrm{CH}_{3}$ signals were observable. (C and $\mathbf{D}$ ) Both relative and absolute signals were higher in the $1 \mathrm{D}$ ge-HSQC (heteronuclear single quantum coherence) as compared with the $1 \mathrm{D}$ ge-HMQC (heteronuclear multiple quantum coherence).

the use of carbon-13-MRS $\left({ }^{13} \mathrm{C}-\mathrm{MRS}\right)$ to study dietary fat uptake and retention in human liver. The low natural abundance of ${ }^{13} \mathrm{C}$, and hence the low incorporation in endogenous lipids, makes ${ }^{13} \mathrm{C}$-enriched fatty acids excellent candidates for in vivo MR tracer studies to study the uptake of dietary fat, metabolism, and net retention (10). Classically, the use of ${ }^{13} \mathrm{C}-\mathrm{MRS}$, however, inherently suffers from low sensitivity when compared with ${ }^{1} \mathrm{H}$-MRS. To overcome this problem of the relative low sensitivity of the ${ }^{13} \mathrm{C}$ nucleus, indirect detection techniques could be explored (11). These techniques selectively detect protons, which are attached to ${ }^{13} \mathrm{C}$ nucleus, via spectral editing; they thereby benefit from the enhanced sensitivity of the ${ }^{1} \mathrm{H}$ signal. Next to this increase in sensitivity, an additional advantage of these techniques is that standard ${ }^{1} \mathrm{H}$ localization techniques can be used, e.g. point resolved spectroscopy (PRESS) (12) and stimulated echo acquistion mode (STEAM) (13), to select a specified volume from which the signal is acquired.

Gradient enhanced heteronuclear multiple-quan-

A $1 \mathrm{D} \mathrm{ge-HMQC}$

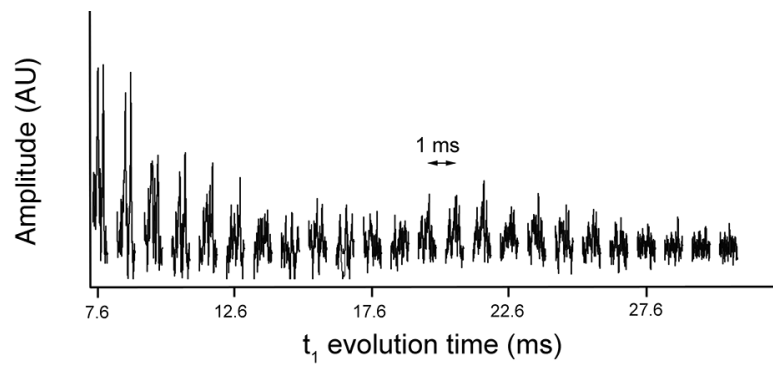

B 1D ge-HSQC

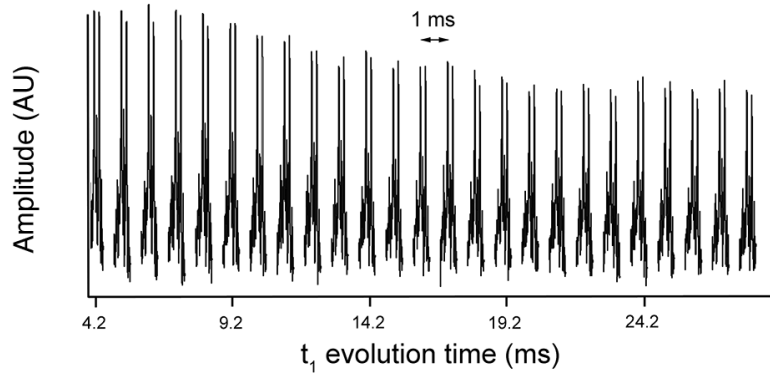
tum coherence spectroscopy (ge-HMQC) and gradient enhanced single-quantum coherence spectroscopy (ge-HSQC) allow the acquisition of ${ }^{1} \mathrm{H}-\left[{ }^{13} \mathrm{C}\right]$ signals while simultaneously spoiling the unwanted ${ }^{1} \mathrm{H}-\left[{ }^{12} \mathrm{C}\right]$ and water signals in a single scan (14). Here, we investigated whether this special class of indirect detection methods, based on the indirect detection of single-quantum ${ }^{13} \mathrm{C}$ coherences, could be used to detect dietary fat retention in human liver. The large chemical shift of the ${ }^{13} \mathrm{C}$ resonances that contribute to the

Figure 2. Spectra acquired from the Intralipid phantom with increasing $t_{1}$ in the $1 D$ ge-HMQC (heteronuclear multiple quantum coherence) and the 10 ge-HSQC (heteronuclear single quantum coherence). (A and $\mathbf{B}) \operatorname{In} \mathbf{A}$, the results for the $1 \mathrm{D}$ ge-HMQC are shown, while in $\mathbf{B}$, the outcome of the $1 \mathrm{D}$ ge-HSQC sequence is presented. The evolution time $\left(t_{1}\right)$ was increased with $1 \mathrm{~ms}$ every step and started at, respectively, 7.6 and $4.2 \mathrm{~ms}$. It is apparent that the signal acquired with the ge-HMQC decays rapidly with an increasing $t_{1}$. 


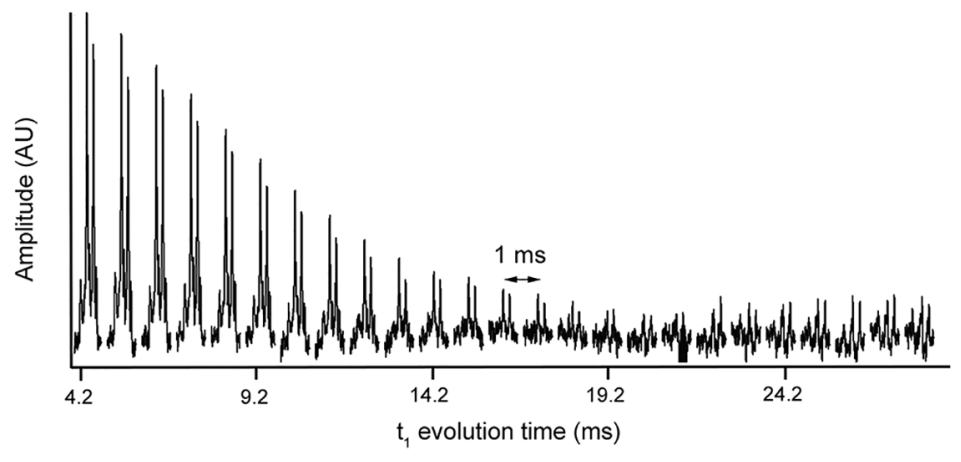

Figure 3. Spectra acquired from the phantom with the uniformly labeled ${ }^{13} \mathrm{C}$ tracer with increasing $\mathrm{t}_{1}$ in the $1 \mathrm{D}$ ge-HSQC (heteronuclear single quantum coherence). The evolution time $\left(\mathrm{t}_{1}\right)$ was increased with $1 \mathrm{~ms}$ every step and started at $4.2 \mathrm{~ms}$. Due to the ${ }^{13} \mathrm{C}-{ }^{13} \mathrm{C}$ couplings in the tracer, the signal now decays rapidly with increasing $t_{1}$.

lipid signal in the ${ }^{1} \mathrm{H}$ spectrum will lead to signal intensity loss and phase distortions when the conventional ge-HMQC and ge-HSQC sequences are applied as 1-dimensional (1D) editing techniques. In this study, we therefore compared 1D ge-HMQC and ge-HSQC sequences, which were specifically designed to refocus the chemical shift in the heteronuclear dimension. We compared the use of the $1 \mathrm{D}$ sequences for the detection of the ${ }^{1} \mathrm{H}-\left[{ }^{13} \mathrm{C}\right]$ lipid signal in phantoms, and we show the feasibility for ${ }^{13} \mathrm{C}$ lipid tracer experiments of the ge-HSQC sequence in the liver in vivo in humans.

\section{Results}

Phantom experiments. In Figure 1, the spectra acquired from the Intralipid phantom with the conventional ge-HMQC and ge-HSQC (Figure 1, A and B, respectively) and with both 1D sequences (Figure 1, C and D) are shown. It is apparent that, in both conventional sequences, the ${ }^{13} \mathrm{C}$ chemical shift evolution during the mixing time leads to phase-distorted lipid signals with decreased signal intensities. The insertion of the ${ }^{13} \mathrm{C}$ inversion pulses in both the ge-HMQC and the ge-HSQC resolves these issues, leading to spectra where both ${ }^{1} \mathrm{H}-\left[{ }^{13} \mathrm{C}\right]$ lipid methyl and methylene signals can be detected unambiguously. Not only was the absolute signal intensity of these $\mathrm{CH}_{2}$ - and $\mathrm{CH}_{3}$-resonances higher with the ge-HSQC, but the calculated ${ }^{13} \mathrm{C}$-enrichment - based on the $\mathrm{CH}_{2}$ intensities - was also higher $(0.72 \%$ with the ge-HMQC versus $0.93 \%$ with the ge-HSQC), indicating less signal loss with the ge-HSQC sequence, as this value is closer to the known natural abundance of ${ }^{13} \mathrm{C}$ isotopes of $1.1 \%$.

In contrast to the PRESS-based ge-HMQC sequence, the proton magnetization is along the longitudinal axis during the quantum coherence evolution period in the STEAM-based ge-HSQC sequence. To investigate the influence of passive ${ }^{1} \mathrm{H}-{ }^{1} \mathrm{H}$ couplings during the evolution time $\left(\mathrm{t}_{1}\right)$ period in the ge-HMQC, the $t_{1}$ evolution period was prolonged stepwise in the next experiment, which is shown in Figure 2. The signal acquired with the $1 \mathrm{D}$ ge-HMQC sequence is rapidly decaying with a longer $t_{1}$, while it is clearly visible that this decay is less pronounced when using the 1D ge-HSQC sequence, which already has an intrinsic shorter minimum $\mathrm{t}_{1}$.

For the in vivo measurements, increased local $\mathrm{B}_{0}$ field variations in tissue are expected when compared with a homogeneous phantom. As these local inhomogeneities can counteract the effect of the applied gradients, stronger gradient dephasing is required. A longer $t_{1}$ period enables the use of gradients with a longer duration and, thus, a larger dephasing area. However, although the ge-HSQC sequence was shown to be insensitive toward ${ }^{1} \mathrm{H}-{ }^{-1} \mathrm{H}$ couplings in the previous experiment with naturally abundance ${ }^{13} \mathrm{C}$, the existence of ${ }^{13} \mathrm{C}-{ }^{13} \mathrm{C}$ couplings in the uniformly labeled fatty acids should be taken into account. This was studied in a next experiment using a phantom with the tracer, which was identical to the tracer that was used for the human experiments, diluted in water. A stepwise increase of $\mathrm{t}_{1}$ led to substantial signal loss, as measured by $1 \mathrm{D}$ ge-HSQC sequence (Figure 3 ). In order to maximize the signal recovery, it is therefore imperative to minimize the $\mathrm{t}_{1}$ evolution time.

In vivo experiments. All results of the in vivo experiments are displayed in Figure 4. Typical examples of a total lipid spectrum ( $\left.{ }^{1} \mathrm{H}-\mathrm{MRS}\right)$ and its concomitant ${ }^{13} \mathrm{C}$ edited lipid spectrum acquired in the liver in vivo are shown (Figure 4, A and B, respectively). Total IHL content in the liver at baseline was $11.3 \pm 3.1 \mathrm{~g} / \mathrm{kg}$ wet weight (ww) for the lean and $42.4 \pm 13.1 \mathrm{~g} / \mathrm{kg}$ ww for the obese subjects $(P=0.06)$. We did not observe significant changes in total IHL content upon meal consumption $(P=0.35$ compared with baseline, Figure 4C). ${ }^{13} \mathrm{C}$ lipid content in the liver did increase significantly in time after the meal $(P<0.01)$, but this increase was not significantly different between the groups $(P=0.29)$, as shown in Figure $4 \mathrm{D}$. Absolute ${ }^{13} \mathrm{C}$ lipid concentrations increased from $0.12 \pm 0.03 \mathrm{~g} / \mathrm{kg}$ ww $(1.1 \%$ of the total lipid content) at baseline to $0.19 \pm$ $0.02 \mathrm{~g} / \mathrm{kg}$ ww after 6 hours in the lean subjects. In the obese subjects, ${ }^{13} \mathrm{C}$ lipid content increased from 0.47 $\pm 0.14 \mathrm{~g} / \mathrm{kg}$ ww at baseline to $0.62 \pm 0.20 \mathrm{~g} / \mathrm{kg}$ ww at 6 hours. 
A

In vivo ${ }^{1} \mathrm{H}$ lipid spectrum

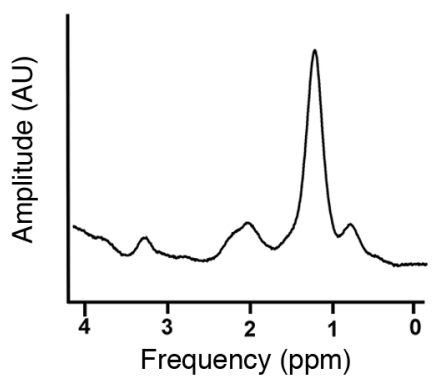

C

Total liver fat content in time

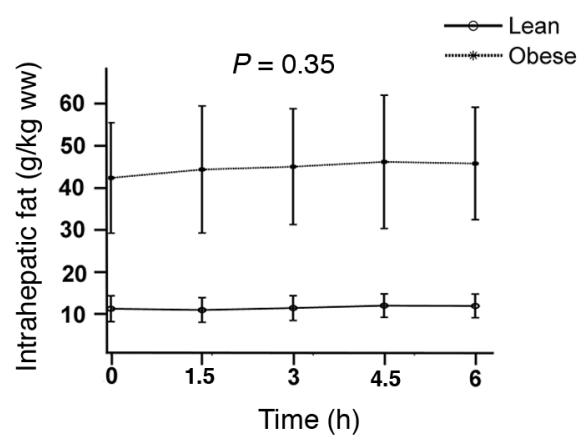

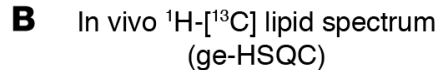

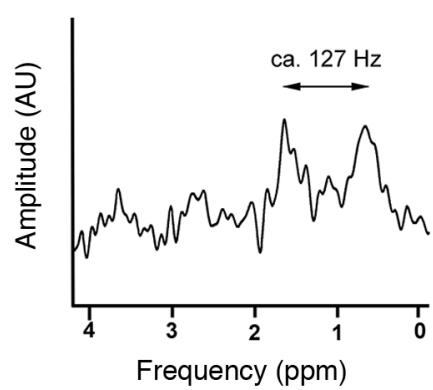

D ${ }^{13} \mathrm{C}$ liver fat content in time

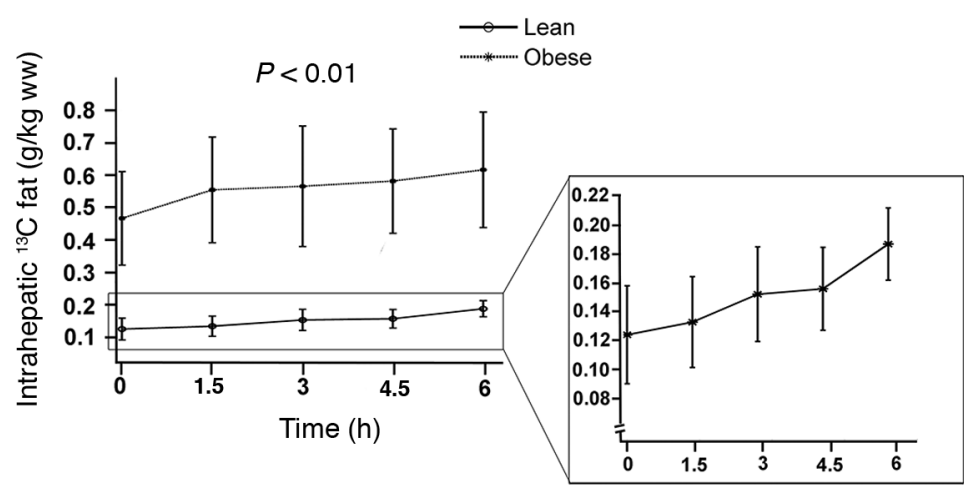

Figure 4. Results from the in vivo experiments. (A and B) Typical example of the total lipid spectrum, acquired with STEAM (stimulated echo acquistion mode) ${ }^{1} \mathrm{H}-\mathrm{MRS}$, and the concomitant ${ }^{13} \mathrm{C}$-edited lipid spectrum, acquired with the ge-HSQC (heteronuclear single quantum coherence) sequence. As no decoupling was applied, two lipid $\mathrm{CH}_{2}$ peaks are visible, which are separated by approximately $127 \mathrm{~Hz}$. (C) The total IHL (intrahepatic lipid) pool (g/kg ww) for both lean $(n=5)$ and obese $(n=6)$ subjects at 1,5, 3.0, 4.5, and 6.0 hours after a high-fat meal. Total IHL did not change after the meal (repeated-measures ANOVA, $P=0.35$ ). (D) The absolute concentration of ${ }^{13} \mathrm{C}$ lipids $\left(\mathrm{g} / \mathrm{kg}\right.$ ww) in the liver is plotted in time, showing retention of ${ }^{13} \mathrm{C}$-labeled fatty acids after the meal (repeated-measures ANOVA, $P<0.01$ ).

As the amount of tracer given was body mass dependent, the absolute concentrations were used to calculate the relative tracer retention in the liver after 6 hours. Tracer retention after 6 hours was similar in both groups $(1.5 \% \pm 0.7 \%$ and $1.6 \% \pm 0.5 \%$ for lean and obese subjects respectively, $P=0.92$, Supplemental Figure 1; supplemental material available online with this article; doi:10.1172/jci.insight.84671DS1).

\section{Discussion}

Here, we have demonstrated the feasibility of tracking dietary fatty acids to the liver noninvasively in vivo with 1D ge-HSQC MRS to determine postprandial hepatic lipid retention. Importantly, this MRS-based approach can be performed taking advantage of ${ }^{13} \mathrm{C}$ as a naturally labeled stable isotope; hence, no radioactive tracers are required. The noninvasive measure of dietary fat uptake after a meal revealed that, in both lean and obese subjects, a substantial amount of dietary fat is retained in the liver. We anticipate that this method will permit noninvasive examination of whether and how postprandial fatty acid storage is affected in patients with a fatty liver and, importantly, also offers a way to discriminate the role of dietary fat retention versus de novo lipogenesis and excessive free fatty acids (FFA) lipolysis from adipose tissue. To date, these determinants could only be estimated by modeling approaches, in studies using multiple tracers and requiring liver biopsies (8), which is not feasible in larger intervention studies. Combining the MRS technique with accurate measures of total lipid content will give valuable insight into fat storage and oxidation - and, thus, turnover — of the IHL pool.

The use of quantum coherence spectroscopy techniques in vivo has not been fully explored in humans so far. In an attempt to optimize the measurement of postprandial lipid handling in the human liver in vivo 
A $1 \mathrm{D}$ ge-HMQC
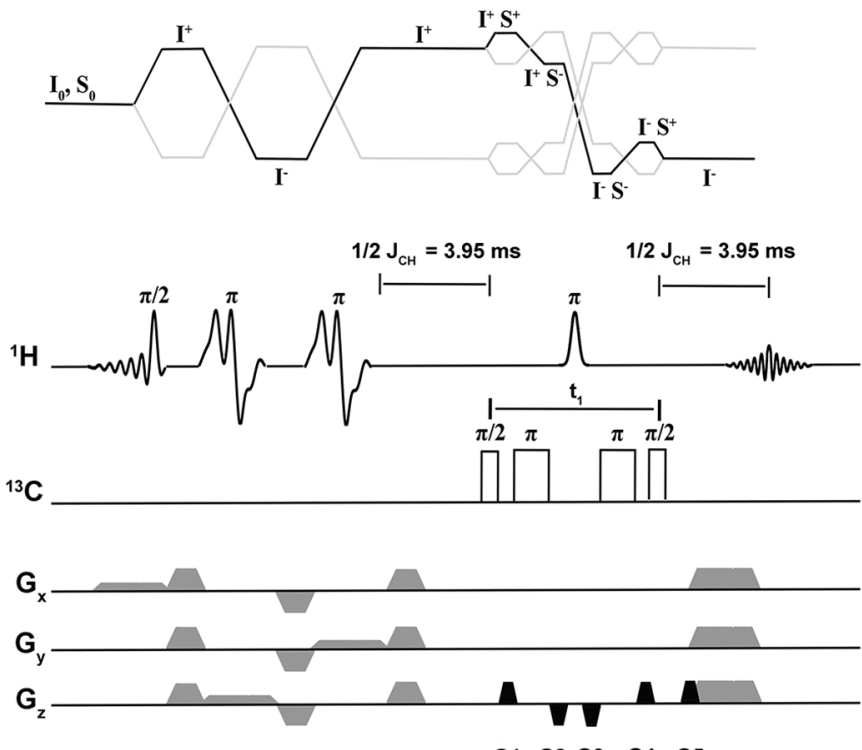

G1 G2 G3 G4 G5
B 1D ge-HSQC
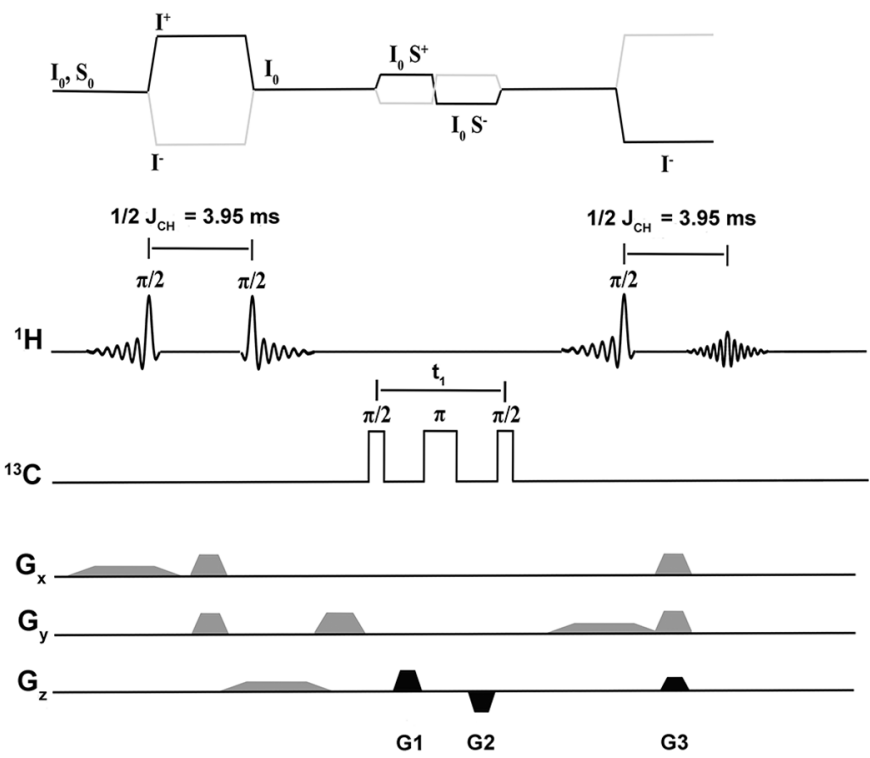

Figure 5. Pulse sequences used in this study. (A) The 1D ge-HMQC (heteronuclear multiple quantum coherence) sequence is shown. The HMQC block was placed after PRESS (point resolved spectroscopy) localization. Two additional ${ }^{13} \mathrm{C}$ inversion pulses were used to refocus the ${ }^{13} \mathrm{C}$ chemical shift evolution. Minimum evolution time $\left(t_{1}\right)$ was 7.6 ms. In contrast to the conventional ge-HMQC sequence, 5 gradients were used for coherence selection. Gradients were applied in a ratio of 1:-1:-1:1:1. (B) The 1D ge-HSQC (heteronuclear single quantum coherence) sequence, based on STEAM (stimulated echo acquistion mode) localization. The inversion pulse during the $t_{1}$, which was used on the ${ }^{1} \mathrm{H}$ channel previously, was now applied on the ${ }^{13} \mathrm{C}$ channel to again refocus ${ }^{13} \mathrm{C}$ chemical shift evolution. Minimum $\mathrm{t}_{1}$ was $4.2 \mathrm{~ms}$, and gradients were applied in a $2:-2: 1$ ratio. In both sequences, $\left.1 / 2\right)_{C H}$ was chosen as $3.95(J=127$ Hz for $\mathrm{CH}_{2}$ lipids). No decoupling was applied.

using a uniformly labeled ${ }^{13} \mathrm{C}$ fatty acid tracer, we have shown here that such techniques offer a robust alternative to subtraction-based J-editing sequences. As gradients are being used for clean coherence selection, quantum coherence sequences offer signals in a single shot, thereby omitting the need for subtraction and hence reducing motion artifacts. Phantom experiments revealed that the addition of ${ }^{13} \mathrm{C}$ inversion pulses to conventional 2D ge-HMQC and ge-HSQC sequences is essential in order to obtain ${ }^{1} \mathrm{H}-\left[{ }^{13} \mathrm{C}\right]$-lipid signals in a 1D spectrum without phase distortions. The higher signal-to-noise ratio (SNR), as obtained with the 1D approach, now offers the opportunity to quantitatively study the dynamic incorporation of meal-derived ${ }^{13} \mathrm{C}$ lipid tracer into the IHL pool in vivo on a $3 \mathrm{~T}$ clinical scanner. As ${ }^{1} \mathrm{H}-{ }^{1} \mathrm{H}$ coupling evolution is absent in the $t_{1}$ period in the ge-HSQC, this sequence yields higher signals when compared with the ge-HMQC.

Previously, direct ${ }^{13} \mathrm{C}$-MRS was explored to investigate dietary fat retention in humans (10). The intrinsically low sensitivity and the large chemical shift dispersion, as encountered in direct ${ }^{13} \mathrm{C}$-MRS, forced the researchers to perform unlocalized measurements. It was reported that approximately $9 \%$ of the ingested ${ }^{13} \mathrm{C}$ tracer was stored in the liver of control subjects at the peak uptake after 6 hours; however, these measurements were performed unlocalized. These numbers are higher than what we report here, but contaminating signals from adipose tissue - as well as from ${ }^{13} \mathrm{C}$ tracer in the stomach and intestine - are likely to affect these measurements and will result in higher uptake levels if unlocalized measurements are performed. Considering the amount of tracer used, the low sensitivity of the direct ${ }^{13} \mathrm{C}$ approach and the limited amount of tracer stored in the liver as indicated by our results further suggest that the successful detection of differences in ${ }^{13} \mathrm{C}$ enrichment in the IHL pool using direct ${ }^{13} \mathrm{C}-\mathrm{MRS}$ is extremely challenging, if not impossible.

Heteronuclear quantum coherence spectroscopy can be seen as a special class of indirect spectroscopy techniques. Indirect ${ }^{13} \mathrm{C}$ spectroscopy, in general, offers higher sensitivity when compared with direct ${ }^{13} \mathrm{C}$-MRS, as the signal of the (more sensitive) protons that are directly attached to a ${ }^{13} \mathrm{C}$ nucleus is acquired. In addition to this, proper localization of the signal into the tissue of interest is possible by using ${ }^{1} \mathrm{H}$ localization techniques. The use of proton-observed carbon-edited (POCE) methodology $(15,16)$, another indirect 
${ }^{13} \mathrm{C}$ spectroscopy method, was recently demonstrated for the detection of the ${ }^{1} \mathrm{H}-\left[{ }^{13} \mathrm{C}\right]$ lipid signal in rats $(17)$. As this J-difference method requires phase cycling to cancel out the very large ${ }^{1} \mathrm{H}-\left[{ }^{12} \mathrm{C}\right]$ signals, it is prone to motion-induced subtraction artifacts, and that becomes a major problem in motion-sensitive tissue such as the liver in humans. In rats measured under anesthesia, motion artifacts due to breathing were absent (17), but initial human experiments in our lab using the same POCE methodology on a clinical 3T scanner indeed revealed that subtraction artifacts as a result of motion posed an insurmountable problem for accurate and robust detection of ${ }^{1} \mathrm{H}-\left[{ }^{13} \mathrm{C}\right]$-lipid signals (data not shown). Therefore, we concluded that the POCE methodology does not lead to a robust and accurate measure of the naturally abundant ${ }^{13} \mathrm{C}$ signal in humans. The 1D ge-HSQC sequence offers a quarter of the maximal theoretical signal when compared with the described methodology in rats, which is a result of the use of STEAM localization and gradient coherence selection. In practice, these differences are much smaller, as the STEAM localization sequence has an intrinsically shorter echo time (TE), and furthermore, the robustness of spoiling the large ${ }^{1} \mathrm{H}-\left[{ }^{12} \mathrm{C}\right]$-lipid (and water) signals with gradients outweighs the remaining signal loss.

In the present study, the use of both the $1 \mathrm{D}$ ge-HMQC and the $1 \mathrm{D}$ ge-HSQC was investigated. Identical to the above described reasoning, the intrinsic TE in the 1D ge-HSQC is shorter than in the 1D ge-HMQC. In practice, the reference signals acquired with both of these sequences were (almost) equal, showing that the theoretical 50\% signal loss in STEAM spectroscopy was balanced by the longer TE due to PRESS localization in the currently compared protocols. Apart from the localization technique, the sequences differ in the quantum coherence evolution period. During the $t_{1}$ period in the ge-HMQC, both ${ }^{1} \mathrm{H}-{ }^{1} \mathrm{H}$ couplings and ${ }^{13} \mathrm{C}-{ }^{13} \mathrm{C}$ couplings are evolving, while the ${ }^{1} \mathrm{H}-{ }^{1} \mathrm{H}$ couplings are absent in the ge-HSQC, as ${ }^{1} \mathrm{H}$ magnetization is stored along the longitudinal axis during mixing time (TM). It was shown in phantom experiments that, as a result of (strong) ${ }^{1} \mathrm{H}-{ }^{1} \mathrm{H}$ coupling of the lipid $\mathrm{CH}_{2}$ signal, the ${ }^{1} \mathrm{H}-\left[{ }^{13} \mathrm{C}\right]$-lipid signal is rapidly decaying with increased $t_{1}$ in the $1 \mathrm{D}$ ge-HMQC. This strong signal decay was absent in the $1 \mathrm{D}$ ge-HSQC. As a larger number of radio frequency (RF) pulses was required to fully refocus ${ }^{13} \mathrm{C}$ chemical shift evolution in the $1 \mathrm{D}$ ge-HMQC, the minimum $t_{1}$ was already increased when compared with the $1 \mathrm{D}$ ge-HSQC. On top of that, a larger number of RF pulses also induced increased signal losses as a consequence of (small) miscalibrations. Taken together, we concluded that the 1D ge-HSQC sequence yields higher absolute signals and also higher (relative) ${ }^{13} \mathrm{C}$-enrichment and is therefore the superior technique for in vivo ${ }^{13} \mathrm{C}$ fatty acid tracking.

As mentioned, ${ }^{13} \mathrm{C}-{ }^{13} \mathrm{C}$ couplings do evolve during the quantum coherence evolution period in the $1 \mathrm{D}$ ge-HSQC. In nature, only $1.1 \%$ of all carbon nuclei is present as ${ }^{13} \mathrm{C}$ isotope, which reduces the chance that two ${ }^{13} \mathrm{C}$ nuclei are directly adjacent virtually to 0 . Therefore, signal losses due to ${ }^{13} \mathrm{C}-{ }^{13} \mathrm{C}$ couplings are negligible when applied to molecules with naturally abundant ${ }^{13} \mathrm{C}$. This situation, however, changes in the case of the uniformly labeled ${ }^{13} \mathrm{C}$ tracer, as we used in the present study, where ${ }^{13} \mathrm{C}$ nuclei are indeed adjacent to each other. As shown in a phantom experiment with the uniformly labeled tracer, the ge-HSQC signal decreases considerably with increasing $\mathrm{t}_{1}$, indicating signal losses due to ${ }^{13} \mathrm{C}-{ }^{13} \mathrm{C}$ couplings. As longer gradients are preferred to completely dephase all residual signals in vivo, a longer $t_{1}$ can be chosen when one is interested in ${ }^{13} \mathrm{C}$ natural abundance only. When uniformly labeled tracers are used, however, short $\mathrm{t}_{1}$ are required and the $\mathrm{t}_{1}$ of $6.1 \mathrm{~ms}$ chosen in this study ensured only minor signal losses due to ${ }^{13} \mathrm{C}-{ }^{13} \mathrm{C}$ couplings, while gradient strength and additional phase cycling were sufficient to spoil the large ${ }^{1} \mathrm{H}-\left[{ }^{12} \mathrm{C}\right]$-lipid signal. The reported ${ }^{13} \mathrm{C}$ enrichment after the meal was not corrected for signal losses due to ${ }^{13} \mathrm{C}-{ }^{13} \mathrm{C}$ couplings, which might lead to a small underestimation of tracer uptake.

The described 1D ge-HSQC methodology was applied to measure the storage of ${ }^{13} \mathrm{C}$-labeled dietary fatty acids in the liver in vivo and was used to quantify the amount of lipids stored from a single meal. We indeed were able to measure a significant increase in ${ }^{13} \mathrm{C}$ lipid content in the liver in both lean and obese subjects, reflecting incorporation of dietary lipids into hepatic liver fat. It is important to note that, with ${ }^{1} \mathrm{H}$-MRS, we are not able to detect any changes in the total IHL content upon the consumption of a meal with the current protocol. This inability is most likely attributed to a lack in sensitivity to detect the very small changes with respect to the large background signal $\left({ }^{12} \mathrm{C}-{ }^{1} \mathrm{H}\right.$ and ${ }^{13} \mathrm{C}-{ }^{1} \mathrm{H}$ lipid signals. This further underlines the strength and added value of the ${ }^{13} \mathrm{C}$ tracer approach to allow the detection of nutritional effects on liver fat accumulation

Approximately $1.5 \%$ of the orally administered tracer could be detected in the liver 6 hours after the meal, and this calculated tracer retention was similar for lean and obese subjects. Although the retention of $1.5 \%$ may seem small, it should be considered that a daily intake of 100 grams of fat, spread over 3 meals, is normal for humans consuming a Western diet, suggesting that 1.5 grams of dietary fat could end up in 
the liver. For a healthy subject with a relatively low liver-fat percentage of $1 \%$, which equals approximately $15 \mathrm{~g}$ of total hepatic lipid, dietary fat could therefore make up around $10 \%$ of the total IHL pool over a day. This is in close agreement with estimations ( $15 \%)$ from the aforementioned study by Donnelly et al., who used liver biopsies (8). This thus suggests that liver fat turnover is high and that the contribution of dietary fat is physiologically relevant. Furthermore, considering that dietary fat retention did not return to premeal levels within 6 hours and meal intervals during the day are around 4-5 hours, these data suggest that dietary fatty acid intake can lead to substantial increases in total liver fat concentrations, hence leading to the development from a healthy to a fatty liver. Therefore, strategies to_influence the storage of dietary fat into the liver may be an effective tool to prevent hepatic steatosis.

To conclude, we have shown here that we were able to acquire $1 \mathrm{D}{ }^{13} \mathrm{C}$-edited spectra in a single shot by using ${ }^{13} \mathrm{C}$ inversion pulses in the quantum coherence evolution period of a ge-HSQC sequence. The $1 \mathrm{D}$ ge-HSQC sequence was applied in vivo, and postprandial handling of dietary fatty acids in the liver could be followed dynamically. This technique allows the performance of in vivo studies to investigate the role of postprandial fatty acid handling in both healthy subjects and subjects at risk for increased liver fat. Additional studies using this noninvasive tool can give insight into how to influence dietary fat retention, for example by varying meal timing and composition, to prevent excessive lipid storage in the liver.

\section{Methods}

\section{MR Sequences and equipment}

Experiments were performed on a 3T clinical MR system (Achieva 3T-X, Philips Healthcare) using a butterfly-loop ${ }^{1} \mathrm{H}$ transmit/receive quadrature coil combined with two $16-\mathrm{cm}{ }^{13} \mathrm{C}$ transmit/receive surface coils in quadrature-mode for optimal ${ }^{1} \mathrm{H}$ receiving sensitivity (Rapid Biomedical $\mathrm{GmbH}$ ).

The 2-pulse sequences that have been compared in this study are displayed in Figure 5. While in conventional 2D ge-HMQC and ge-HSQC sequences, the chemical shift of the heteronucleus and determines the acquired phase in the quantum coherence $t_{1}$, this frequency-dependent phase modulation is unwanted in $1 \mathrm{D}$ spectroscopy because of signal intensity losses and phase distortions in the ${ }^{1} \mathrm{H}-\left[{ }^{13} \mathrm{C}\right]$ spectrum. Signal losses and phase distortions will be most pronounced for metabolites with large chemical shifts of ${ }^{13} \mathrm{C}$ resonances, as is the case for the $\mathrm{CH}_{2}$ resonances of lipid (resonances over a range of $\sim 20 \mathrm{ppm}$ ). In both $1 \mathrm{D}$ sequences, the ${ }^{13} \mathrm{C}$ chemical shift is refocused by adding $180^{\circ} \mathrm{RF}$ pulses in the quantum coherence evolution time. In contrast to the conventional $2 \mathrm{D}$ ge-HMQC and ge-HSQC sequences, the $\mathrm{t}_{1}$ is kept constant.

\section{$1 D$ ge-HMQC}

The 1D ge-HMQC sequence starts with conventional PRESS localization, which is followed by a time delay of $1 / 2 \mathrm{~J}_{\mathrm{CH}}\left(3.95 \mathrm{~ms}\right.$ for lipid $\left.\mathrm{CH}_{2}\right)$ to produce nonobservable antiphase magnetization for the protons attached to a ${ }^{13} \mathrm{C}$ nucleus (18). The first $90^{\circ}$ pulse on the ${ }^{13} \mathrm{C}$ channel then generates zero and double quantum coherences (ZQC and DQC, respectively). For the highlighted coherence pathway in Figure $1 \mathrm{~A}$, the DQCs are converted to ZQCs by the first ${ }^{13} \mathrm{C} 180^{\circ} \mathrm{RF}$ pulse, then converted back to DQC again by the ${ }^{1} \mathrm{H} 180^{\circ}$ pulse, followed by another conversion to ZQCs by the second ${ }^{13} \mathrm{C} 180^{\circ}$ pulse. The last $90^{\circ}$ ${ }^{13} \mathrm{C}$ pulse converts the coherence states back to antiphase magnetization, which evolves to observable transverse magnetization after the $1 / 2 \mathrm{~J}_{\mathrm{CH}}$ delay. The symmetric sequence created not only refocuses ${ }^{13} \mathrm{C}$ chemical shift evolution, but also prevents the $\mathrm{B}_{0}$ inhomogeneity-induced delayed echo formation of the ${ }^{1} \mathrm{H}-\left[{ }^{13} \mathrm{C}\right]$ signal (19). Due to the creation of four different coherence states during the $\mathrm{t}_{1}, 5$ instead of 3 are applied to achieve coherence selection. A gradient ratio of G1:G2:G3:G4:G5 = 1:1:-1:-1:1.006 was used for selection of the multiple quantum coherences. Because all gradients can be of equal strength, the maximal gradient strength is used to efficiently dephase all unwanted coherences. Gradients $(30 \mathrm{mT} / \mathrm{m})$ of equal duration $\left(1.0 \mathrm{~ms}\right.$ ) were applied in the $\mathrm{Z}$ direction only. The ${ }^{13} \mathrm{C} R \mathrm{R}$ pulses were all played out as hard pulses with a duration of $0.34 \mathrm{~ms}\left(90^{\circ}\right)$ and $0.67 \mathrm{~ms}\left(180^{\circ}\right)$ respectively. Duration of the Gaussian shaped ${ }^{1} \mathrm{H} 180^{\circ} \mathrm{RF}$ pulse was $1.09 \mathrm{~ms}$. This led to a minimum $\mathrm{t}_{1}$ of $7.6 \mathrm{~ms}$. The first ${ }^{13} \mathrm{C}$ pulse was phase cycled to improve spoiling of unwanted coherences, and the offset of the ${ }^{13} \mathrm{C}$ pulses was set at the midchain ${ }^{13} \mathrm{C}-\mathrm{CH}_{2}$ resonance ( $\left.29 \mathrm{ppm}\right)$. To minimize the total TE, the PRESS spoiler gradient areas were chosen in a ratio of $1:-1: 1: 3$, with the largest gradient placed in the last $1 / 2 \mathrm{~J}_{\mathrm{CH}}$ delay. The total TE of the sequence was $28.9 \mathrm{~ms}$. 


\section{D ge-HSQC}

STEAM localization lies at the basis of the $1 \mathrm{D}$ ge-HSQC sequence (Figure 1B), as was reported previously $(19,20)$. The delay of $1 / 2 \mathrm{~J}_{\mathrm{CH}}$ after the ${ }^{1} \mathrm{H}$ excitation $\mathrm{RF}$ pulse leads to antiphase magnetization of the protons attached to a ${ }^{13} \mathrm{C}$ nucleus. The second ${ }^{1} \mathrm{H} 90^{\circ} \mathrm{RF}$ pulse converts the transversal antiphase magnetization into longitudinal magnetization. During the TM, a hard ${ }^{13} \mathrm{C} 90^{\circ} \mathrm{RF}$ pulse results in the formation of a single quantum coherence state, which will evolve based on the ${ }^{13} \mathrm{C}$ resonance frequency. A next hard ${ }^{13} \mathrm{C} 180^{\circ} \mathrm{RF}$ pulse, which is placed exactly in the middle of the $t_{1}$, changes the sign of the coherence order (from +1 to -1 ). A final hard ${ }^{13} \mathrm{C} 90^{\circ} \mathrm{RF}$ pulse again creates longitudinal antiphase magnetization. A crusher gradient (duration of $4.44 \mathrm{~ms}$, strength $30 \mathrm{mT} / \mathrm{m}$ ) is used in the TM period prior to the ${ }^{13} \mathrm{C} 90^{\circ} \mathrm{RF}$ pulses to dephase any existing transversal magnetization. The third and final ${ }^{1} \mathrm{H}$ $90^{\circ} \mathrm{RF}$ pulse flips back the longitudinal magnetization to the transversal plane, which will then generate an observable echo after another delay of $1 / 2 \mathrm{~J}_{\mathrm{CH}}$. Gradients were used in the ratio of G1:G2:G3= $2:-2: 1.006$. At this point, only $50 \%$ of the maximal available gradient strength could be used for dephasing of the unwanted coherences. The strength of the gradients in the $t_{1}$ period was $30 \mathrm{mT} / \mathrm{m}$, and the gradient in the refocusing period was set to $15.087 \mathrm{mT} / \mathrm{m}$. These coherence selection gradients were applied in the $\mathrm{Z}$ direction. For the phantom experiments, the duration of these gradients was set to 1.5 $\mathrm{ms}$, while for the in vivo experiments, the gradients were lengthened to $2.5 \mathrm{~ms}$. As for the ge-HMQC, the length of the ${ }^{13} \mathrm{C} \mathrm{RF}$ pulses was $0.34 \mathrm{~ms}$ for the $90^{\circ}$ and $0.67 \mathrm{~ms}$ for the $180^{\circ}$ pulse, and the pulses where applied with an offset around $29 \mathrm{ppm}$. The minimum $\mathrm{t}_{1}$ was $4.2 \mathrm{~ms}$ for the phantom experiments and 6.1 $\mathrm{ms}$ for the in vivo experiments. The TM period was $22.6 \mathrm{~ms}$ and $24.5 \mathrm{~ms}$, respectively, while total TE of the sequence was, by definition, equal to $1 / \mathrm{J}_{\mathrm{CH}}$ (7.9 $\mathrm{ms}$ for the lipid $\mathrm{CH}_{2}$ resonance).

For the phantom experiments, where the ge-HMQC and ge-HSQC methods were compared, phase cycling of the ge-HSQC was identical to the ge-HMQC sequence. Hence, the phase of the first ${ }^{13} \mathrm{C}$ pulse $(\mathrm{x},-\mathrm{x})$ and the acquisition channel $(\mathrm{x},-\mathrm{x})$ were cycled. As the phantom experiment showed that the 1D ge-HSQC sequence was superior for the current aim, only the 1D ge-HSQC sequence was applied in the in vivo experiments. To further improve the spoiling of unwanted echoes in the in vivo experiments, additional phase cycling was applied to the proton excitation pulse and the second ${ }^{13} \mathrm{C}$ pulse of the HSQC block (16 steps phase cyling).

\section{Phantom experiments}

Phantoms. In this study, phantom experiments were performed for two different purposes. In the first experiments, the signal intensities of the 1D ge-HMQC and the 1D ge-HSQC sequence were compared on a 500-ml phantom filled with 20\% Intralipid (Fresenius Kabi Global), a stable fat emulsion of fine droplets of soybean oil. Identical to the situation in vivo, $1.1 \%$ of all carbon nuclei are ${ }^{13} \mathrm{C}$ isotopes (natural abundance). In a subsequent experiment, a spherical phantom with uniformly ${ }^{13} \mathrm{C}$-labeled algal lipids $(1.5 \mathrm{~g})$ dissolved in water was used ( $>98 \%{ }^{13} \mathrm{C} ; 53 \%$ palmitic acid, $28 \%$ oleic acid, $9 \%$ palmitioleic acid, and $6 \%$ linoleic acid; Cambridge Isotopes Laboratories Inc.). Here, the influence of the ${ }^{13} \mathrm{C}-{ }^{13} \mathrm{C}$ couplings in the uniformly labeled fatty acid tracer was tested by increasing the $t_{1}$ of the $1 \mathrm{D}$ ge-HSQC sequence. The same algal lipid mixture was used for the in vivo experiments.

$M R$ spectroscopy. Scout images were acquired to enable placement of a voxel of $20 \times 40 \times 40 \mathrm{~mm}$ in the center of the phantom. The threshold ratio (TR) of all experiments was set to 2,000 ms, and spectra were acquired with a bandwidth of $2,000 \mathrm{~Hz}\left(1,024\right.$ data points). Both the ${ }^{1} \mathrm{H}$ and the ${ }^{13} \mathrm{C}$ pulses were calibrated by measuring a single slice with a varying flip angle. Lipid reference signals were obtained by turning off the coherence selection gradients and by setting the frequency of the ${ }^{13} \mathrm{C} R F$ pulses far off resonance. Shimming was performed using second order FASTMAP-based shimming (21).

First, spectra were acquired with both the conventional ge-HMQC and ge-HSQC sequence - without the added ${ }^{13} \mathrm{C} 180^{\circ}$ pulses (number of spectral acquisitions $[\mathrm{NSA}]=128$ ). In a next experiment, the principle of the $1 \mathrm{D}$ sequences was tested by now turning on these ${ }^{13} \mathrm{C} 180^{\circ}$ pulses. This also allowed comparison between the signal intensities of the 1D ge-HMQC and the 1D ge-HSQC sequence. We also tested the effect of an increased $t_{1}$ in both sequences by measuring 25 spectra with a stepwise increasing $t_{1}$. The starting $t_{1}$ was set to the minimum possible (7.6 and $4.2 \mathrm{~ms}$, respectively), and $t_{1}$ was increased with $1 \mathrm{~ms}$ per spectrum. Each spectrum consisted of 8 averaged acquisitions.

As the ${ }^{13} \mathrm{C}$ nuclei are directly adjacent to each other in the uniformly ${ }^{13} \mathrm{C}$-labeled fatty acids, additional signal losses during $\mathrm{t}_{1}$ are expected due to (strong) ${ }^{13} \mathrm{C}-{ }^{13} \mathrm{C}$ scalar couplings. To be able to choose the appro- 
priate $t_{1}$ for the in vivo experiments, an identical experiment with a series of spectra with increasing $t_{1}$, as was performed on the Intralipid, was also performed on the algal lipid mixture phantom. This last experiment was only done with the $1 \mathrm{D}$ ge-HSQC sequence.

Data analysis. All spectra were analyzed and fitted in the time domain by using the nonlinear leastsquares AMARES algorithm in the jMRUI software package (22). Spectra were apodized with a $5 \mathrm{~Hz}$ Gaussian lineshape. The $\mathrm{CH}_{2}$ peaks of the lipid signal were fitted with a Gaussian lineshape. Because no decoupling was applied, 2 peaks of equal amplitude and with equal line width were fitted for the ${ }^{13} \mathrm{C}$-edited spectra. The ${ }^{13} \mathrm{C}$-enrichment was calculated by dividing the sum of the area of the 2 peaks in the edited spectra with the area under the peak in the reference spectra. A correction factor of 2 was applied to correct for the intrinsic 50\% signal loss as encountered due to the coherence gradient selection.

\section{In vivo experiments}

Subjects. To validate our methodology in humans in vivo, we included 5 lean ( 2 female $/ 3$ male, age $49 \pm 13$ years, and BMI $22 \pm 3 \mathrm{~kg} / \mathrm{m}^{2}$ ) and 6 obese ( 3 female $/ 3$ male, age $52 \pm 10$ years, and BMI $31 \pm 6 \mathrm{~kg} / \mathrm{m}^{2}$ ) subjects. All subjects were considered healthy and weight stable (fluctuations in body mass $<3 \mathrm{~kg}$ ) for at least 6 months prior to the study.

Study protocol. On the test day, the subjects arrived at the university at 7 am after an overnight fast. Two days prior to the test days, subjects were asked to refrain from strenuous physical activity and to consume their habitual diet. After the baseline MRS measurement, subjects consumed a high-fat (60E\% fat, 36E $\%$ carbohydrates and $4 \mathrm{E} \%$ protein) liquid meal, with a total energy content of $753 \mathrm{kcal}$ and fat content of 50.3 g. On top of this, the subject consumed capsules containing $0.1 \mathrm{~g} / \mathrm{kg}$ body weight of the $\mathrm{U}-{ }^{13} \mathrm{C}$ algal lipid mixture. The algal lipid mixture was tested and packaged for oral intake by the clinical pharmacy of the Radboud University Medical Center (Nijmegen, The Netherlands). Subjects drank water ad libitum during the test day. Following on from the liquid meal with tracer, MRS measurements were performed after 1.5, $3.0,4.5$, and 6.0 hours to measure the ${ }^{13} \mathrm{C}$ enrichment in the IHL pool.

MR Spectroscopy. Subjects were positioned in the MRI scanner in supine position and head first. The surface coil was placed over the right hypochondrium and was positioned to ensure that the liver was in the region of the sensitivity of the coil, which was verified with MR imaging. Scout images were acquired prior to the MR spectroscopy protocol, to accurately position the voxel of $40 \times 60 \times 60 \mathrm{~mm}$ inside the liver. Special care was taken to ensure that the voxel was positioned at the very same location during the repeated measurements. A pressure-sensitive sensor was used to keep track of the breathing rhythm and to gate the acquisition of the spectra. Start of the spectroscopy sequence was adjusted per subject and was between 1,000 and 1,500 ms after the top of inspiration.

After placement of the voxel, the ${ }^{1} \mathrm{H}$ pulse angle was calibrated with a standard available algorithm on the Philips MR scanner. Based on the ${ }^{1} \mathrm{H}$ settings, the ${ }^{13} \mathrm{C}$ pulse angle was calibrated according to a calibration curve, which was measured in a phantom beforehand. Following the calibration of the pulses, second-order FASTMAP shimming was performed.

Then, water (NSA $=32$ ) and lipid reference signals (NSA $=64$ ) were acquired by turning off the coherence selection gradients and by setting the frequency of the ${ }^{13} \mathrm{C} R \mathrm{~F}$ pulses far off resonance. For the lipid reference spectrum, the water peak was suppressed using a frequency-selective adiabatic inversion pulse, played out $300 \mathrm{~ms}$ prior to the excitation pulse. The 1D ge-HSQC sequence was started successively. A total of 320 individual acquisitions was averaged to come to the ${ }^{13} \mathrm{C}$-edited lipid spectrum. Total duration of the in vivo protocol was approximately 45 minutes.

Data analysis. Baseline IHL content was calculated from the water and lipid spectra after individual phasing of the individual spectra. For one of the obese subjects, data from the time point at 6.0 hours was excluded due to insufficient quality of the spectra. All spectra were fitted in jMRUI software using Gaussian line shapes. For the ${ }^{13} \mathrm{C}$ edited spectra, the fitting procedure was identical to the procedures described for the phantom experiments. The ratio of the line width of the 2 peaks in the edited spectra and the line width of the peak in the lipid reference scan were kept constant for the repeated measurement in one subject.

Both total fat content, as determined with ${ }^{1} \mathrm{H}-\mathrm{MRS}$, and ${ }^{13} \mathrm{C}$ fat content, as determined with ge-HSQC, are expressed in absolute concentrations (in $\mathrm{g} / \mathrm{kg}$ wet weight) using water as the internal reference and based on previously published assumptions on water content and relaxation times $(1,23)$. As the amount of tracer consumed was different between subjects (based on the body weight of the subject), tracer retention was calculated as a percentage of the total intake of tracer. An average liver mass of $1.5 \mathrm{~kg}$ was used (24) for these calculations. 


\section{Statistics}

All statistical analyses were performed with SPSS Statistics 20 (IBM Corp. Released 2011. Armonk, New York, USA.). Subject characteristics are expressed as mean $\pm \mathrm{SD}$, while all other results are expressed as mean \pm SEM.

For the in vivo experiments, repeated-measures ANOVA was performed to test for differences in total lipid and ${ }^{13} \mathrm{C}$ lipid content in the liver. A 2-tailed independent samples Student $t$ test was performed to test for differences in tracer uptake (in $\%$ of total dose) between groups. A $P$ value less than 0.05 was considered to be statistically significant for all tests.

\section{Study approval}

Experiments were performed at the Maastricht University Medical Center, and the study was approved by the Medical Ethics Committee of the University Hospital Maastricht/Maastricht University (METC azM /UM, Maastricht). Written informed consent was obtained from the subjects prior to the experiments.

\section{Author Contributions}

LL, CIN, JEW, MKCH, PS, and VBSH designed the research study. LL and PAVE conducted the experiments and acquired and analyzed the data. RADG supported and advised on the development of the MR sequence. LL, MKCH, PS, and VBSH discussed and interpreted the results. LL wrote the manuscript. RADG, CIN, MKCH, JEW, PS, and VBSH reviewed and edited the manuscript.

\section{Acknowledgments}

The project was funded by TI Food and Nutrition, a public-private partnership on precompetitive research in food and nutrition. The public partners are responsible for the study design, data collection and analysis, decision to publish, and preparation of the manuscript. The private partners have contributed to the project through regular discussion.

VBSH is supported by a VENI (grant 91611136) for innovative research from the Netherlands Organization for Scientific Research (NWO).

Address correspondence to: Patrick Schrauwen, Department of Human Biology and Human Movement Sciences, Maastricht University Medical Center, PO Box 616, 6229 ER Maastricht, Netherlands. Phone: 31.0.43.388.1502; E-mail: P.Schrauwen@maastrichtuniversity.n1.

CIN's current address is: Philips HealthCare, Best, Netherlands.

1. Szczepaniak LS, et al. Measurement of intracellular triglyceride stores by $\mathrm{H}$ spectroscopy: validation in vivo. Am J Physiol. 1999;276(5 Pt 1):E977-E989.

2. Boesch C, Slotboom J, Hoppeler H, Kreis R. In vivo determination of intra-myocellular lipids in human muscle by means of localized 1H-MR-spectroscopy. Magn Reson Med. 1997;37(4):484-493.

3. Hwang $\mathrm{JH}$, et al. Increased intrahepatic triglyceride is associated with peripheral insulin resistance: in vivo MR imaging and spectroscopy studies. Am J Physiol Endocrinol Metab. 2007;293(6):E1663-E1669.

4. Korenblat KM, Fabbrini E, Mohammed BS, Klein S. Liver, muscle, and adipose tissue insulin action is directly related to intrahepatic triglyceride content in obese subjects. Gastroenterology. 2008;134(5):1369-1375.

5. Seppälä-Lindroos A, et al. Fat accumulation in the liver is associated with defects in insulin suppression of glucose production and serum free fatty acids independent of obesity in normal men. J Clin Endocrinol Metab. 2002;87(7):3023-3028.

6. Targher G, Day CP, Bonora E. Risk of cardiovascular disease in patients with nonalcoholic fatty liver disease. $N$ Engl J Med. 2010;363(14):1341-1350.

7. Reddy JK, Rao MS. Lipid metabolism and liver inflammation. II. Fatty liver disease and fatty acid oxidation. Am J Physiol Gastrointest Liver Physiol. 2006;290(5):G852-G858.

8. Donnelly KL, Smith CI, Schwarzenberg SJ, Jessurun J, Boldt MD, Parks EJ. Sources of fatty acids stored in liver and secreted via lipoproteins in patients with nonalcoholic fatty liver disease. J Clin Invest. 2005;115(5):1343-1351.

9. Labbé SM, et al. Organ-specific dietary fatty acid uptake in humans using positron emission tomography coupled to computed tomography. Am J Physiol Endocrinol Metab. 2011;300(3):E445-E453.

10. Ravikumar B, et al. Real-time assessment of postprandial fat storage in liver and skeletal muscle in health and type 2 diabetes. Am J Physiol Endocrinol Metab. 2005;288(4):E789-E797.

11. de Graaf RA, Rothman DL, Behar KL. State of the art direct $13 \mathrm{C}$ and indirect $1 \mathrm{H}-[13 \mathrm{C}] \mathrm{NMR}$ spectroscopy in vivo. A practical guide. NMR Biomed. 2011;24(8):958-972.

12. Bottomley PA. Spatial localization in NMR spectroscopy in vivo. Ann N Y Acad Sci. 1987;508:333-348. 
13. Frahm J, Bruhn H, Gyngell ML, Merboldt KD, Hänicke W, Sauter R. Localized high-resolution proton NMR spectroscopy using stimulated echoes: initial applications to human brain in vivo. Magn Reson Med. 1989;9(1):79-93.

14. Ruiz-Cabello J, Vuister GW, Moonen CT, van Gelderen P, Cohen JS, van Zij1 PC. Gradient-enhanced heteronuclear correlation spectroscopy: theory and experimental aspects. 1992. J Magn Reson. 2011;213(2):446-466.

15. de Graaf RA, Brown PB, Mason GF, Rothman DL, Behar KL. Detection of [1,6-13C2]-glucose metabolism in rat brain by in vivo 1H-[13C]-NMR spectroscopy. Magn Reson Med. 2003;49(1):37-46.

16. Rothman DL, et al. 1H-Observe/13C-decouple spectroscopic measurements of lactate and glutamate in the rat brain in vivo. Proc Natl Acad Sci U S A. 1985;82(6):1633-1637.

17. Jonkers RA, Geraedts TR, van Loon LJ, Nicolay K, Prompers JJ. Multitissue assessment of in vivo postprandial intracellular lipid partitioning in rats using localized 1H-[13C] magnetic resonance spectroscopy. Magn Reson Med. 2012;68(4):997-1006

18. Yahya A, Allen PS. Single-shot 3D localized indirect 13C detection. Presented at: 13th Annual ISMRM Scientific Meeting and Exhibition; May 20, 2004; Kyoto, Japan. Abstract 680.

19. Knuttel A, Kimmich R, Spohn KH. Indirect 13C tomography and volume-selective spectroscopy via proton NMR. I. Spectrocopic techniques. J Magn Reson. 1990;86(3):526-541.

20. de Graaf RA, De Feyter HM, Rothman DL. High-sensitivity, broadband-decoupled (13) C MR spectroscopy in humans at 7T using two-dimensional heteronuclear single-quantum coherence. Magn Reson Med. 2015;74(4):903-914

21. Gruetter R. Automatic, localized in vivo adjustment of all first- and second-order shim coils. Magn Reson Med. 1993;29(6):804-811.

22. Vanhamme L, van den Boogaart A, Van Huffel S. Improved method for accurate and efficient quantification of MRS data with use of prior knowledge. J Magn Reson. 1997;129(1):35-43.

23. Guiu B, et al. Quantification of liver fat content: comparison of triple-echo chemical shift gradient-echo imaging and in vivo proton MR spectroscopy. Radiology. 2009;250(1):95-102.

24. Molina DK, DiMaio VJ. Normal organ weights in men: part II-the brain, lungs, liver, spleen, and kidneys. Am J Forensic Med Pathol. 2012;33(4):368-372. 